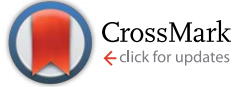

Cite this: J. Mater. Chem. A, 2016, 4, 529

Received 17th August 2015

Accepted 25th November 2015

DOI: $10.1039 / c 5 t a 06472 d$

www.rsc.org/MaterialsA

\section{Efficient identification of hydrophobic MOFs: application in the capture of toxic industrial chemicals $\dagger$}

\author{
Peyman Z. Moghadam,,$^{\mathrm{a}}$ David Fairen-Jimenez $\ddagger^{\mathrm{b}}$ and Randall Q. Snurr*a
}

Water is an ever-present component in the air, and competitive adsorption of water is a major challenge in many applications of adsorbents, including capture of toxic industrial chemicals (TICS) from the atmosphere. For metal-organic framework (MOF) adsorbents, the presence of water often leads to major material instabilities that could limit their practical performance. MOFs displaying hydrophobic behavior might be useful in overcoming these problems. In this work, we present a new computational strategy to quickly identify hydrophobic MOFs based on their water Henry's constants. Starting with a database of 137953 hypothetical MOFs, we identified 45975 structures as hydrophobic based on their simulated water Henry's constants. Using grand canonical Monte Carlo simulations, we further analyzed 2777 of these hydrophobic materials whose linkers did not contain chemical functionalization. The results show insignificant water uptake in the identified MOFs, confirming their hydrophobic nature. The capability of the hydrophobic MOFs was assessed for ammonia capture under humid conditions, and analysis of the data generated from this high-throughput computational screening revealed the role of the textural properties and surface chemistry on the removal of toxic compounds. The results suggest that if materials are too hydrophilic, they adsorb too much water and show little or no selectivity towards TICs. On the other hand, if they are too hydrophobic, they adsorb too little ammonia.

\section{Introduction}

Water adsorption is one of the most important characteristics of porous metal-organic frameworks (MOFs) ${ }^{1-3}$ because the coadsorption of water can greatly affect the ability to selectively adsorb a target species. Capture of volatile toxic industrial chemicals (TICs) is a potential application of MOFs where competitive water adsorption is a particular challenge. ${ }^{4}$ TIC capture has historically been centered on adsorption and trapping by activated carbon. ${ }^{5-7}$ While activated carbon is clearly useful, its capacity is lower than desired and its ability to capture low-molecular-weight chemicals such as ammonia, $\mathrm{NO}_{x}$, and formaldehyde is somewhat limited. ${ }^{5}$ For example, maximal $\mathrm{NH}_{3}$ adsorption capacity is $130 \mathrm{mg} \mathrm{g}^{-1}$ for highly activated carbons impregnated with $\mathrm{H}_{2} \mathrm{SO}_{4} \cdot{ }^{8}$ The relatively low capacity of carbons is a consequence of their ill-defined

\footnotetext{
${ }^{a}$ Department of Chemical and Biological Engineering, Northwestern University, Evanston, Illinois 60208, USA. E-mail: snurr@northwestern.edu

${ }^{b}$ Department of Chemical Engineering and Biotechnology, University of Cambridge, Pembroke St., Cambridge CB2 3RA, UK

$\dagger$ Electronic supplementary information (ESI) available: Force field models, Henry's constant values for selected MOFs, experimental water adsorption isotherms, ammonia and water uptakes for hydrophobic MOFs, and top candidate structures with ammonia uptake higher than $6 \mathrm{~mol} \mathrm{~kg}^{-1}$. See DOI: 10.1039/c5ta06472d

\$ These authors contributed equally to this work.
}

porosity, less than optimal pore and channel dimensions, and weak adsorbate-adsorbent interactions with these compounds. ${ }^{4}$ Therefore, capturing chemical agents requires novel adsorbents featuring specific characteristics such as strong adsorption sites to create very high gravimetric and volumetric adsorption capacities. Furthermore, structures with synthetically tunable cavities are highly desired since they allow for structural design for optimal capacity and selectivity.

MOFs are, in principle, capable of satisfying these requirements. The tunability of the pore textural properties (i.e. pore surface area, volume, size, and shape) as well as surface chemistry (i.e. functional groups) allows for generation of an almost limitless number of MOFs and the ability to tailor their features for separation applications. ${ }^{9-12}$ A number of MOFs have been examined in the literature for the removal of TICs from air with both experiments and molecular simulation. ${ }^{\mathbf{1 3 - 1 6}}$ Numerous reports have discussed detrimental water effects on MOF's adsorption performance..$^{15,17-21}$ Indeed, an inherent challenge in the capture of TICs in humid conditions is the competitive adsorption of water from the atmosphere. In order to produce optimal MOF adsorbents, it is therefore desirable to design porous structures that have high affinity for TICs but not for water. This suggests that hydrophobic materials might be a good starting point. A number of studies in the literature have focused on different strategies to increase MOF hydrophobicity - and stability with respect to water vapor - by 
introducing hydrophobic moieties such as fluorinated functional groups or shielding the metal clusters with bulky functional groups. ${ }^{22-27}$ However, the principles for designing hydrophobic MOFs and the effects of textural properties on hydrophobicity are not well understood.

Molecular simulations can provide insights into water adsorption in MOFs, but equilibrating water isotherms using grand canonical Monte Carlo (GCMC) simulations is notoriously tedious and time consuming. ${ }^{28}$ These simulations require a large number of Monte Carlo steps, as slight changes in water arrangements result in drastic energy changes in the system and low acceptance rates of the Monte Carlo moves. Moreover, performing experimental water adsorption tests for the thousands of known MOF structures is not feasible. To address the above challenges, we present a fast and efficient computational approach involving calculation of Henry's constants to predict the water adsorption capabilities of a large number of adsorbents, and we apply it to identify hydrophobic structures in a database of 137953 MOFs. ${ }^{29}$ We also determine the affinities of ammonia and methane, as representative polar and nonpolar molecules, in these MOFs and compare them with the adsorption affinity of water. As a complement to the Henry's constant calculations, which are relevant at very low loadings, the performance of the selected hydrophobic MOFs for water adsorption and ammonia capture at finite loading is tested using GCMC simulations.

\section{Approach: Henry's constant $\left(K_{\mathrm{H}}\right)$ calculations for efficient screening}

The shape of an adsorption isotherm provides a great deal of information about the interactions present in a system ${ }^{30}$ and, in the case of water adsorption, about the hydrophilic or hydrophobic character of the material. Water in hydrophobic MOFs exhibits Type $\mathrm{V}$ adsorption isotherms, which indicate weak water-MOF interactions, with low loadings at low pressures followed by water condensation in the pores at higher pressures due to strong water-water interactions. Examples of Type V isotherms in MOFs include water adsorption in ZIF-8, ${ }^{31}$ $\mathrm{Zn}$ (pyrazol), ${ }^{32}$ and $\mathrm{Al}(\mathrm{NDC}) .^{33}$ In contrast, hydrophilic MOFs such as HKUST-1 and MOF-74 exhibit Type I isotherms and adsorb large amounts of water at low pressure as a consequence of strong water-MOF interactions, which are due to the presence of open metal sites in the case of HKUST-1 and MOF74..$^{31,34-36}$ Regardless of their classification, adsorption isotherms can be interpreted in simple terms by plotting them on a log-log scale as illustrated in Fig. 1. For all isotherms, the low pressure regime can be described by a Henry's constant $\left(K_{\mathrm{H}}\right)$, identified in this $\log -\log$ representation by a slope of 1 . In a standard representation, $K_{\mathrm{H}}$ is the slope of the isotherm in the Henry region at very low loadings and is a simple way to quantify the adsorbate-adsorbent interaction affinity. At the highest pressures, the saturation capacity of a given adsorbate is determined by the available pore volume $\left(V_{\mathrm{p}}\right)$ and the density of the adsorbed fluid. As shown in Fig. 1, the difference between Type I and Type V isotherms is the deviation of the isotherm

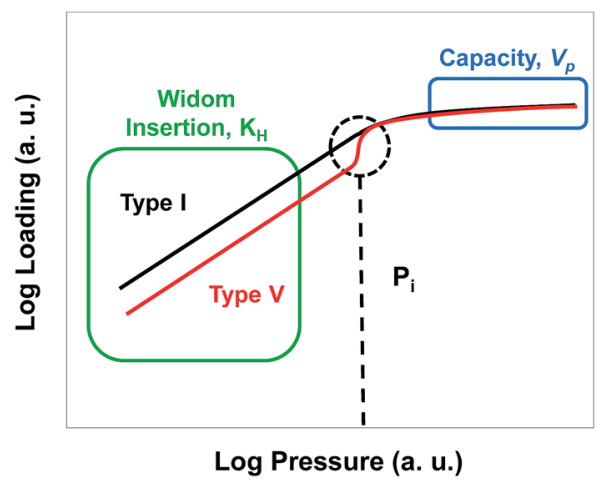

Fig. 1 Schematic showing different regions of Type I and $V$ adsorption isotherms on a log-log scale.

from linearity as the pressure increases; for Type I isotherms the slope becomes less than unity due to pore saturation, and for Type $\mathrm{V}$ isotherms the slope becomes greater than unity due to a cooperative adsorption effect at a given pressure which we will call $P_{\mathrm{i}}$. Ghosh et al. ${ }^{28}$ suggested that the hydrophobicity of MOFs could be quantified by the pressure at which water condenses in the pores, with a higher pressure indicating a more hydrophobic MOF. However, calculating or measuring the full isotherm is very time consuming. We hypothesized that $P_{\mathrm{i}}$ could be correlated with the more easily calculated $K_{\mathrm{H}}$, and $K_{\mathrm{H}}$ therefore could be used as a metric to estimate the hydrophilicity or hydrophobicity of a given MOF.

Henry's constant can be obtained from the low pressure regime of a simulated or experimental adsorption isotherm. However, using this approach is not efficient for screening a large number of structures, especially for water adsorption. Alternatively, $K_{\mathrm{H}}$ can be computed by using the Widom insertion method. ${ }^{37}$ In this method, the adsorbate molecule is inserted in the adsorbent at randomly chosen positions and its energy is calculated each time before it is removed from the system. By repeating the process over a large number of random points, it is possible to quickly evaluate the guest-MOF interaction without including the contribution of guest-guest interactions. ${ }^{38}$ The benefit of this method is that the calculations are orders of magnitude faster than calculating water adsorption isotherms using GCMC simulations. Fig. S1† compares the Henry's constants obtained from the slope of the isotherm at low pressure from GCMC simulations with those computed from the Widom method for water and methane in a number of selected MOFs. Given the excellent agreement observed between the two methods, we decided to carry out all other $K_{\mathrm{H}}$ calculations in this work using the Widom insertion method.

\section{Simulation details}

Adsorbate-adsorbent and adsorbate-adsorbate interactions were modeled with a Lennard-Jones (LJ) plus Coulomb potential with a LJ cut-off distance of $12.8 \AA$ and no tail corrections. Electrostatic interactions were computed using the Ewald summation method for both adsorbate-adsorbent and 
adsorbate-adsorbate interactions. The force field parameters for water were taken from the TIP4P ${ }^{39}$ model. The TraPPE force field was used for ammonia ${ }^{40}$ and methane. ${ }^{41}$ All adsorbate force field parameters are listed in the ESI. $\dagger$ The force field parameters and partial charges for ZIF-8, Al(NDC), and Zn-pyrazole are described in our previous publication. ${ }^{28}$ The LJ parameters for the framework atoms of all other MOFs were taken from the Universal Force Field (UFF). ${ }^{42}$ For FMOF-1, the partial charges on $\mathrm{CF}_{3}$ groups were taken from the work of Dalvi et $a .^{43}$ The partial charges for all other atoms in FMOF-1 were obtained from DFT calculations (see ESI $\dagger$ ). The partial charges for MIL-47 were obtained from the work of Yazaydin et al. ${ }^{44}$ and those for the hypothetical MOFs were calculated from the extended charge equilibrium method. ${ }^{45}$ All MOFs were treated as rigid in the simulations.

Henry's constants were computed using the Widom insertion method. We used orientational-biasing to insert the adsorbate molecules at positions throughout the simulation cell. We first compared results with 10000,100000 , and 1000000 insertions in MIL-47, ZIF-8, Al(NDC), and Zn-pyrazole, and found that 100000 provided sufficient accuracy (see Fig. S1 $\dagger$ ). For the screening of the hypothetical MOFs, we therefore used 100000 insertions. The amount adsorbed for water and ammonia at finite loading was calculated using grand canonical Monte Carlo (GCMC) simulations ${ }^{46}$ implemented in the RASPA molecular simulation software. ${ }^{47}$ The Monte Carlo moves attempted were insertions, deletions, displacements, and rotations plus, for binary mixtures of water and ammonia, identity changes. We used $1 \times 10^{5}$ cycles for equilibration and another $1 \times 10^{5}$ cycles for production in the ammonia pure component simulations. For water simulations (pure and mixtures), we used at least $4 \times 10^{5}$ cycles each for the equilibrium and production periods. A cycle is defined as the maximum of 20 or the number of molecules in the system. The number of unit cells in each MOF was adjusted to be at least twice the LJ cut-off distance.

\section{Results and discussion}

TICs present in the air are generally found as trace amounts with very low partial pressures, where adsorption is in the Henry's law region of the adsorption isotherms. Lab-scale experiments for ammonia capture often use a partial pressure of $c a .290 \mathrm{~Pa}$, for example. ${ }^{48,49}$ For hydrophobic MOFs, water adsorption before condensation is also in the Henry's law region. In this case, the selectivity of a TIC over water can be estimated simply by the ratio of the individual $K_{\mathrm{H}}$ values. ${ }^{50} \mathrm{We}$ initially calculated and compared the $K_{\mathrm{H}}$ values for water, ammonia as a representative TIC, and methane as a representative non-polar molecule for a small number of MOFs with different levels of hydrophobicity: Al-NDC, MIL-47, Zn-pyrazole, and ZIF-8. Using the same force field parameters as used here, Ghosh et al. simulated water uptake in Al-NDC, Zn-pyrazole, and ZIF-8 and found good agreement between experimental and simulated water adsorption isotherms. ${ }^{28}$ Fig. 2 shows the $K_{\mathrm{H}}$ values for water, ammonia, and methane in the selected MOFs, as well as the selectivity of ammonia and methane over
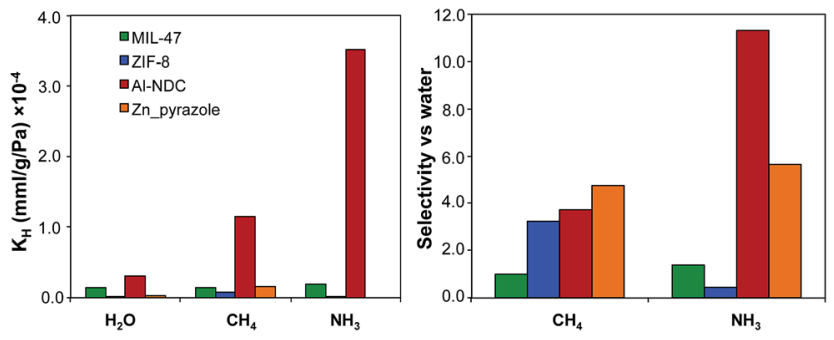

Fig. 2 (Left) Henry's constants $\left(K_{H}\right)$ for water, methane, and ammonia and (right) selectivities for methane and ammonia over water for the four selected MOFs.

water as calculated by the ratio of their Henry's constants. Surprisingly, among the hydrophobic MOFs studied, the structure with the highest affinity for water (i.e. Al-NDC, the least hydrophobic) presents high selectivities for ammonia and methane. Furthermore, the MOF with the lowest affinity for water (i.e. ZIF-8, the most hydrophobic) shows the lowest selectivity for ammonia and moderate selectivity for methane. One question that arises is whether this observed trend can be generalized towards a larger number of hydrophobic MOFs and to what extent the relative hydrophobicity in MOFs can provide preferential adsorption towards a specific toxic chemical. To answer this question, we calculated $K_{\mathrm{H}}$ and the selectivities of ammonia and methane over water for a larger number of hydrophobic and hydrophilic MOFs.

First, we wanted to validate our approach for discriminating between different levels of hydrophobicity in MOFs via their water Henry's constants. We had previously simulated water adsorption isotherms in Al-NDC, MIL-47, Zn-pyrazole, and ZIF-8 and compared them with experiments where available. ${ }^{28}$ In addition to these MOFs, we simulated the water adsorption isotherm for FMOF-1 (Fig. S2 $\dagger$ ), a superhydrophobic MOF with fully fluorinated pores. ${ }^{26,51}$ Predicted water isotherms for these 5 MOFs (Fig. 3a) show that water condenses at different pressures for the different MOFs, indicating a range of water affinities. Note that in FMOF-1, water does not condense in the pores even at $100 \% \mathrm{RH}$ in agreement with experimental measurements. ${ }^{26}$ Fig. 3 b shows that the water condensation $P_{\mathrm{i}}$ inversely correlates
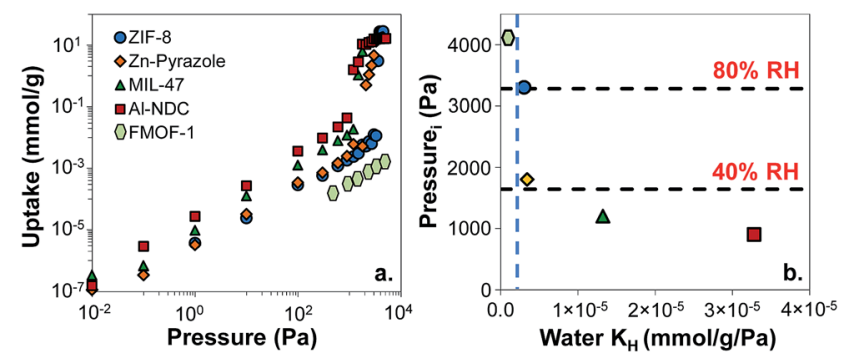

Fig. 3 (a) Simulated water adsorption isotherms for five MOFs studied at 298 K. (b) Water condensation pressures $P_{\mathrm{i}}$ versus Henry's constants $\left(K_{\mathrm{H}}\right)$. The blue dashed line represents the $K_{\mathrm{H}}=5 \times 10^{-6} \mathrm{mmol} \mathrm{g}^{-1} \mathrm{~Pa}^{-1}$ threshold criterion for hydrophobicity. Light green hexagons, FMOF-1; blue circles, ZIF-8; orange diamonds, Zn-pyrazole; green triangles, MIL-47; red squares, Al-NDC. 
with water $K_{\mathrm{H}}$. For example, the superhydrophobic MOF, FMOF-1, has the lowest water $K_{\mathrm{H}}\left(\right.$ i.e. $\left.2 \times 10^{-7} \mathrm{mmol} \mathrm{g}^{-1} \mathrm{~Pa}^{-1}\right)$ and the highest $P_{\mathrm{i}}$ (the $P_{\mathrm{i}}$ for FMOF-1 is set to be equal to the water saturation vapor pressure). For ZIF-8, condensation occurs around $80 \%$ RH. Zn-pyrazole, Al-NDC, and MIL-47 exhibit relatively less hydrophobic behavior, as the condensation steps occur at ca. 40\%, 30\%, and $20 \% \mathrm{RH}$, respectively.

For TIC capture, we consider a scenario where the relative humidity is $80 \%$ in the atmosphere. Under these conditions, we want a MOF to be hydrophobic enough that only a very small amount of water is adsorbed. Using ZIF-8 as a benchmark, we will consider MOFs to be hydrophobic if their water $K_{\mathrm{H}}$ values are lower than $5 \times 10^{-6} \mathrm{mmol} \mathrm{g}^{-1} \mathrm{~Pa}^{-1}$, shown by the vertical, blue, dashed line in Fig. 3b. To support this choice, we collected experimental water isotherms for another 19 MOFs from the literature (Fig. S4†). ${ }^{27,35,52-56}$ From the selected MOFs, 16 have Type $\mathrm{V}$ water isotherms and three have Type I isotherms (Fig. S4 $\dagger$ ). From the experimental isotherms, we calculated the condensation pressure $P_{\mathrm{i}}$ and the water Henry's constants and plotted $P_{\mathrm{i}}$ versus $K_{\mathrm{H}}$ (Fig. S3 $\dagger$ ). If our criterion of $K_{\mathrm{H}}<5 \times 10^{-6}$ mmol g ${ }^{-1} \mathrm{~Pa}^{-1}$ for hydrophobic MOFs is reasonable, then any MOF with $K_{\mathrm{H}}$ less than this value should have a value of $P_{\mathrm{i}}$ of at least $80 \%$ RH. Similarly, MOFs with $K_{\mathrm{H}}>5 \times 10^{-6} \mathrm{mmol} \mathrm{g}^{-1} \mathrm{~Pa}^{-1}$ should have a value of $P_{\mathrm{i}}$ less than $80 \%$ RH. Fig. S3† shows that this is, indeed, true for the 19 MOFs for which we could find experimental water isotherms, thus supporting our proposed $K_{\mathrm{H}}$ threshold for hydrophobicity. Note that very hydrophilic MOFs such as HKUST-1 (ref. 35) and Mg-MOF-74 (ref. 56) have water $K_{\mathrm{H}}$ values orders of magnitude higher than the proposed $K_{\mathrm{H}}$ threshold, with values larger than $0.05 \mathrm{mmol} \mathrm{g}^{-1} \mathrm{~Pa}^{-1}$.

After validation of our $K_{\mathrm{H}}$ criterion, we applied it to a database of MOFs with 137953 hypothetical structures previously developed in our group. ${ }^{29}$ Given the very large number of structures, it is not practical to calculate water adsorption isotherms for every structure and examine their water affinities. On the other hand, by calculating water $K_{\mathrm{H}}$ using the Monte Carlo Widom insertion method we can rapidly screen the database for hydrophobic MOFs. Following this approach, we calculated the water $K_{\mathrm{H}}$ for all 137953 structures in the database, and by implementing the $K_{\mathrm{H}}$ criterion described above we identified 45975 hydrophobic hypothetical MOFs. To analyze their selectivity, we also calculated the $K_{\mathrm{H}}$ values for ammonia and methane and compared them with those obtained for water.

The hypothetical MOF database contains not only structures with a wide range of textural properties (i.e. pore size, surface area, and pore volume) but also contains a diverse surface chemistry due to the presence of different functional groups. In order to investigate first the effects of textural properties on structure-property relationships, we studied the 2777 nonfunctionalized hydrophobic structures present in the database. Fig. 4 shows the selectivity values for ammonia and methane over water versus the pore volume for the hydrophobic MOFs (i.e. water $K_{\mathrm{H}}<5 \times 10^{-6} \mathrm{mmol} \mathrm{g}^{-1} \mathrm{~Pa}^{-1}-$ Fig. $4 \mathrm{a}$ and b) and 3372 unfunctionalized MOFs with somewhat higher affinities for water (i.e. water $K_{\mathrm{H}}<50 \times 10^{-6} \mathrm{mmol} \mathrm{g}^{-1} \mathrm{~Pa}^{-1}-\mathrm{Fig}$. $4 \mathrm{c}$ and d). For both groups, the color code in Fig. 4 represents the $K_{\mathrm{H}}$ values for water. In general, MOFs with pore volumes larger
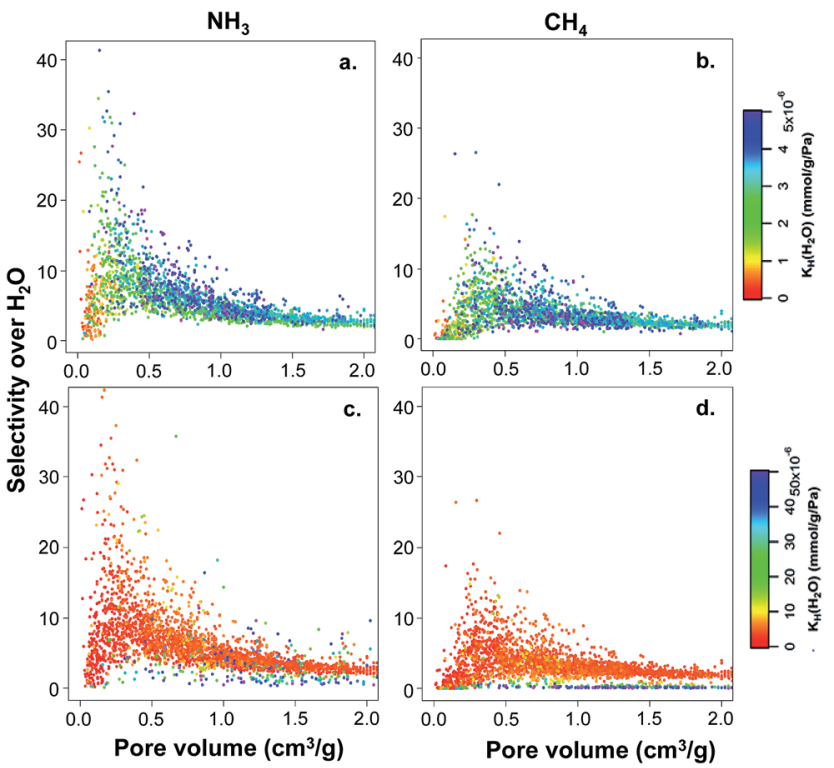

Fig. 4 Calculated ammonia and methane selectivity over water as a function of pore volume for (a) and (c) ammonia and (b) and (d) methane for non-functionalized MOFs at $298 \mathrm{~K}$. The graphs in (a) and (b) are for the 2777 non-functionalized hydrophobic MOFs, whereas the graphs in (c) and (d) are for 3372 non-functionalized MOFs as described in the text. The color code represents water $K_{H}$ values; note the different scales in the upper and lower graphs. Every point in the graphs is a different MOF structure.

than $1 \mathrm{~cm}^{3} \mathrm{~g}^{-1}$ exhibit very low selectivities towards both ammonia and methane. In contrast, MOFs with lower pore volume can exhibit either low or high TIC selectivity. The differences found in the selectivity of these lower pore volume MOFs are related to their hydrophobic/hydrophilic character. In particular, MOFs with only moderate hydrophobicity (i.e. $K_{\mathrm{H}}$ values between $2 \times 10^{-6}$ and $4 \times 10^{-6} \mathrm{mmol} \mathrm{g}^{-1} \mathrm{~Pa}^{-1}$, green to blue in Fig. 4a and b) exhibit high TIC selectivity, whereas highly hydrophobic MOFs with very low water $K_{\mathrm{H}}$ (red to yellow in Fig. 4a and b) do not show high selectivity. This is more prominent for ammonia than methane. MOFs with higher affinities for water (i.e. MOFs with larger water $K_{\mathrm{H}}$ shown by darker colors in Fig. 4c and d) show low TIC selectivity, suggesting that competitive water adsorption is important in these MOFs.

Fig. 5 shows the relationship between TIC selectivity and other textural properties, namely the pore size and pore volume, for the non-functionalized hydrophobic MOFs. The pore size is characterized here by the largest cavity diameter. ${ }^{57}$ The highest selectivity is achieved when the largest cavity diameter is ca. $4 \AA$, which is comparable to the kinetic diameter of $\mathrm{CH}_{4}$ $(3.8 \AA)$ and $\mathrm{NH}_{3}(3.6 \AA)^{58}$ vs. the smaller $\mathrm{H}_{2} \mathrm{O}(2.6 \AA) .{ }^{\mathbf{1 0 5 9}}$ As illustrated by the color codes in Fig. 5 , the pore size and pore volume are interrelated parameters. Note that with a $4 \AA$ largest cavity diameter, only one methane or ammonia molecule can fit across the pore. MOFs containing pores of up to $c a .8 \AA$ diameter, i.e. with the possibility of adsorbing a double layer of ammonia and methane, show rather good selectivities with higher pore volumes. 


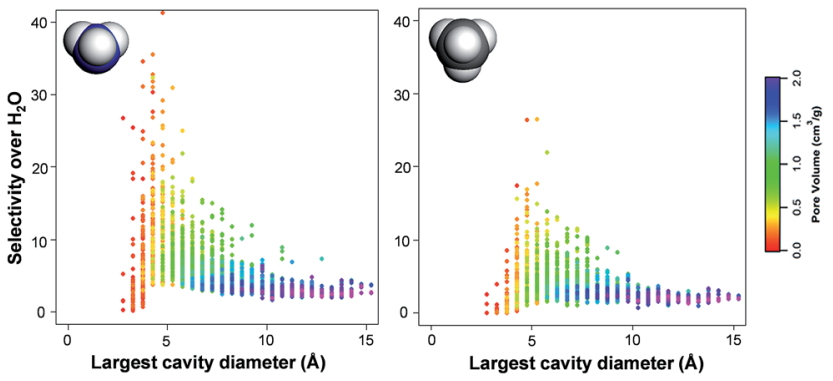

Fig. 5 Calculated ammonia and methane selectivity over water as a function of the MOF's largest cavity diameter for (left) ammonia and (right) methane for non-functionalized hydrophobic MOFs at $298 \mathrm{~K}$. The color code represents the MOF's pore volume.

Following the textural property analysis, we assessed the effects of surface chemistry on ammonia and methane selectivity. We classified the 45975 hydrophobic MOFs present in the database into five different categories depending on the functional groups present: (i) non-functionalized MOFs; (ii) polar groups (i.e. $-\mathrm{NH}_{2},-\mathrm{OH}$, and $-\mathrm{CN}$ ); (iii) alkyl; (iv) ether; and (v) halogens. Fig. 6 shows this classification as well as the resulting frequency of occurrence for each category versus the selectivities.

In general, the structures with halogens show the lowest ammonia and methane selectivity. In the case of methane, frameworks with non-functionalized structures also exhibit low selectivities. Frameworks containing alkyl groups can show low selectivities, but also the highest selectivity towards methane and ammonia. These longer functional groups affect TIC selectivity by a combination of decreasing the pore size and being more hydrophobic. No clear trends were evident for other functional groups due to the dependency of selectivity on a combination of factors that arise from both structural properties as well as the presence and the density of the functional groups.

In the results above, 2777 non-functionalized hydrophobic MOFs were identified from the hypothetical MOF database
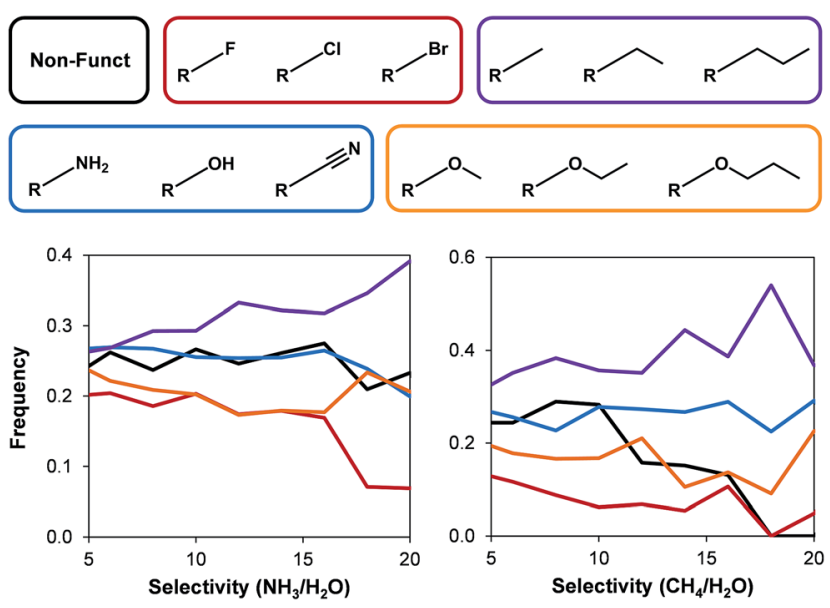

Fig. 6 The effects of different functional groups on (left) ammonia and (right) methane selectivity over water for all hydrophobic hypothetical MOFs.
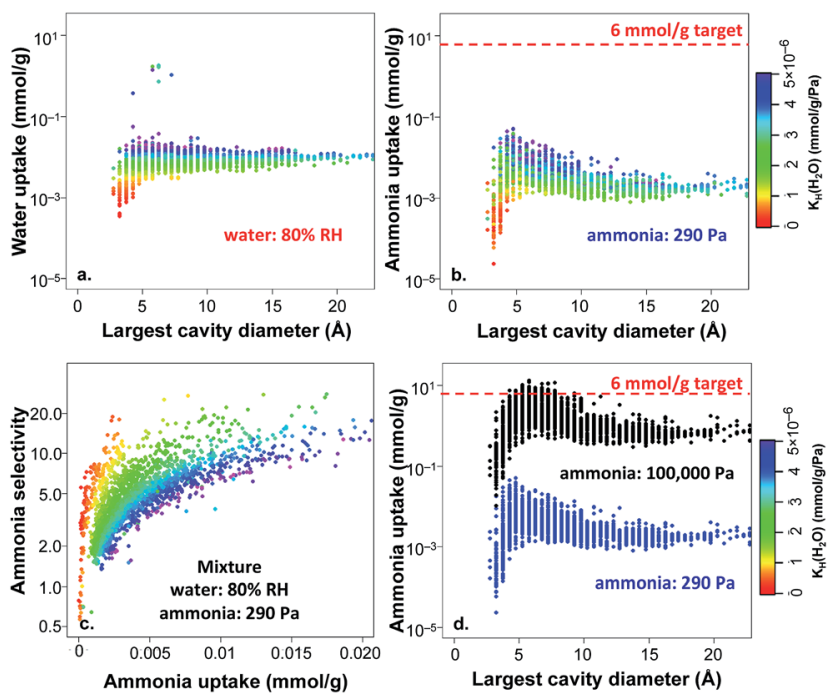

Fig. 7 Simulated adsorption amounts for (a) pure-component water at $80 \% \mathrm{RH}$, (b) pure-component ammonia at $290 \mathrm{~Pa}$, (c) selectivity of ammonia over water for a binary mixture of ammonia at $290 \mathrm{~Pa}$ and water $80 \% \mathrm{RH}$, (d) pure-component ammonia at $290 \mathrm{~Pa}$ and 100000 $\mathrm{Pa}$. All simulations were performed for 2777 unfunctionalized hydrophobic MOFs at $298 \mathrm{~K}$

based on the water $K_{\mathrm{H}}$ criterion, and the selectivities were all estimated based on the ratio of Henry's constants. To further explore these materials, we performed GCMC simulations for the adsorption of ammonia, water, and their mixtures, focusing on conditions of $80 \% \mathrm{RH}$, i.e. a water partial pressure of $3280 \mathrm{~Pa}$. Fig. 7a shows the water uptake versus the largest cavity diameter for adsorption of pure water at $3280 \mathrm{~Pa}$. Since the MOFs were selected based on the satisfaction of our defined hydrophobicity criterion, the majority of the MOFs in Fig. 7a exhibit very low water uptake (i.e. less than $0.1 \mathrm{mmol} \mathrm{g}^{-1}$ ) even at $80 \% \mathrm{RH}$. Notably, the water adsorption correlates very well with the calculated water Henry's constants as illustrated by the color coding in Fig. 7a and not with the pore size. The small magnitude of water adsorption provides further confidence in the hydrophobic nature of the studied MOFs and the computational strategy we used to define hydrophobicity in MOFs.

As discussed above, for practical applications it is necessary to capture trace amounts of TICs at very low partial pressures from the atmosphere, and competitive water adsorption needs to be minimized. Aiming to evaluate ammonia adsorption in the identified unfunctionalized hydrophobic MOFs, we also performed GCMC simulations for pure-component ammonia at $290 \mathrm{~Pa}$, corresponding to the partial pressure commonly used in breakthrough experiments. ${ }^{48,49}$ As shown in Fig. 7b, ammonia adsorption under dry conditions (i.e. pure-component ammonia) is quite low and does not exceed $0.1 \mathrm{mmol} \mathrm{g}^{-1}$ even for optimal pore sizes of $c a$. 4-5 $\AA$; none of the hydrophobic MOFs meet the target of $6 \mathrm{mmol} \mathrm{g}^{-1}$ (ref. 60) for ammonia capture under these conditions. Interestingly, the ammonia uptake is also correlated with the water $K_{\mathrm{H}}$ values (color coding): the more hydrophobic a MOF is, the less affinity it has towards ammonia. 
Fig. 7c shows the ammonia selectivity for a binary mixture of ammonia and water as a function of ammonia uptake at $290 \mathrm{~Pa}$ of ammonia and $80 \% \mathrm{RH}$. The selectivity is defined as:

$$
S_{\mathrm{NH}_{3} / \mathrm{H}_{2} \mathrm{O}}=\frac{x_{\mathrm{NH}_{3}} / x_{\mathrm{H}_{2} \mathrm{O}}}{y_{\mathrm{NH}_{3}} / y_{\mathrm{H}_{2} \mathrm{O}}}
$$

where $x_{i}$ denotes the mole fraction of component $i$ in the adsorbed phase and $y_{i}$ the mole fraction in the gas phase. Selectivity values greater than unity mean that ammonia is more strongly adsorbed than water. For MOFs with water $K_{\mathrm{H}}<1 \times 10^{-6}$ mmol $\mathrm{g}^{-1} \mathrm{~Pa}^{-1}$, although the selectivity can be as high as 20 , ammonia uptake is quite low since the majority of these MOFs have small pores. As the water $K_{\mathrm{H}}$ values become larger and approach $5 \times 10^{-6} \mathrm{mmol} \mathrm{g}^{-1} \mathrm{~Pa}^{-1}$, high selectivity as well as relatively higher ammonia uptakes are attained. As shown in Fig. S5, $\uparrow$ the mixture simulations predict rather similar uptakes for either ammonia or water in comparison with their purecomponent adsorption amounts at the same partial pressures, indicating no significant co-adsorption effects for either component under these conditions. In general, and in correlation with the results shown in Fig. 4 and 5 based on $K_{\mathrm{H}}$ values, the results in Fig. 7 confirm that if the structures are too hydrophobic, they are not good candidates for the capture of ammonia under dry or humid conditions.

In order to test if the selected MOFs are capable of reaching the $6 \mathrm{mmol} \mathrm{g}^{-1}$ target for ammonia capture at a higher pressure, we also performed GCMC ammonia simulations at $100000 \mathrm{~Pa}$ ( 1 bar) and compared the results with those obtained at $290 \mathrm{~Pa}$. As shown in Fig. 7d, the ammonia uptake is much higher under these conditions, with 97 hydrophobic MOFs above the $6 \mathrm{mmol} \mathrm{g}^{-1}$ target. At this high pressure, the peak
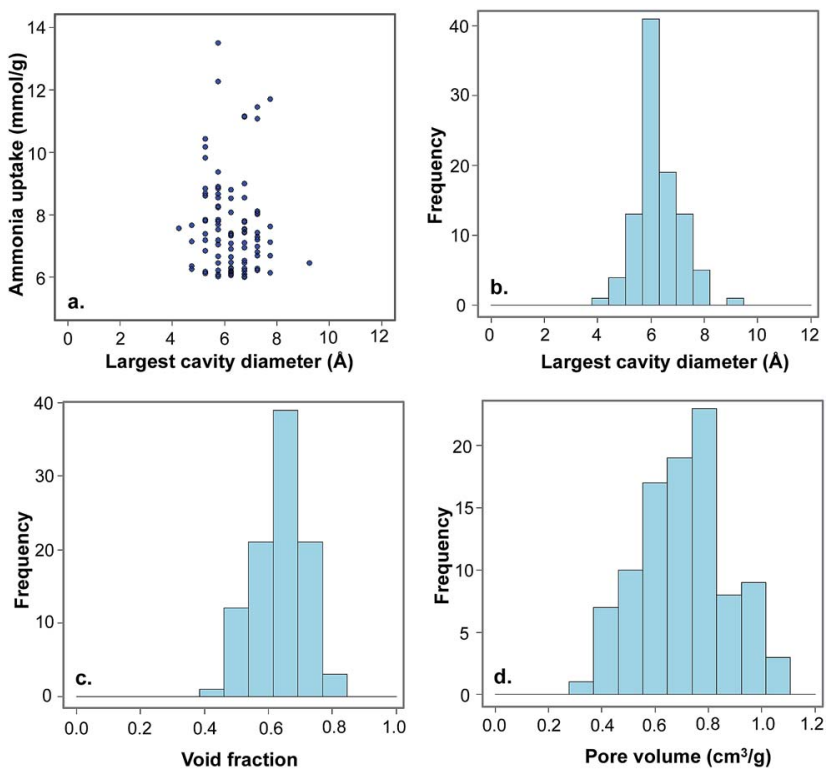

Fig. 8 Structure-property relationships derived for the top 97 MOF candidates with ammonia uptake greater than $6 \mathrm{mmol} \mathrm{g}^{-1}$ at 100000 $\mathrm{Pa}$ and 298 K. (a) Ammonia uptake vs. largest cavity diameter. Histograms of structural properties of the top 97 MOFs are plotted for (b) largest cavity diameter, (c) void fraction, and (d) pore volume. in ammonia uptake is slightly shifted towards MOFs with larger pores because more ammonia molecules are able to fill the additional adsorption sites at higher pressure. The frequency of different structural properties are shown in Fig. 8 for the top 97 MOFs, in which the optimal textural properties are observed for MOFs with pore sizes between 5-7.5 $\AA$, void fractions of $0.6-0.7$, and pore volumes in the range of $0.6-0.8 \mathrm{~cm}^{3} \mathrm{~g}^{-1}$. A number of top MOF structures along with their constituent building blocks are shown in Fig. S6.†

\section{Conclusions}

We presented a new computational strategy based on Henry's constants to quickly identify hydrophobic MOFs and applied it to identify 45975 hydrophobic materials from a pool of 137953 hypothetical MOFs. The Henry's constants also allowed the efficient calculation of the adsorption selectivity for toxic industrial chemicals (TICs) and other molecules in competitive adsorption with water. GCMC simulations of water adsorption at $80 \%$ relative humidity corroborated the existence of little water adsorption in the subset of 2777 unfunctionalized hydrophobic MOFs, providing further proof of the hydrophobic nature of the identified MOFs and the reliability of our method. The selected MOFs were also studied for methane and ammonia capture as representative non-polar and polar molecules. The simulations results show that, on the one hand, strongly hydrophilic MOFs present high competitive water adsorption and therefore exhibit poor selectivity towards TICs. On the other hand, MOFs that are too hydrophobic present low affinity for the TICs and therefore exhibit low selectivity as well. However, MOFs with moderate hydrophobicity and pore sizes comparable to the TIC's kinetic diameter deliver the highest selectivities over water. Investigation of the surface chemistry effects revealed that structures containing alkyl groups present high TIC selectivity due their high hydrophobicity as well as pore size effects.

\section{Acknowledgements}

We thank the Army Research Office (grant W911NF-12-1-0130) and the EPSRC IAA Partnership Development Award (RG/75759) for financial support. Computational work was partly supported by Northwestern University's shared computer system, Quest (project: P20261). D. F.-J. thanks the Royal Society for funding through a University Research Fellowship. We also thank Prof. Omar Yaghi and Dr Hiroyasu Furukawa for supplying the experimental water isotherms for some of the MOFs studied in this work. We thank Dr Pritha Ghosh and Dr Diego A. GómezGualdrón for fruitful discussions.

\section{References}

1 H. Li, M. Eddaoudi, M. O'Keeffe and O. M. Yaghi, Nature, 1999, 402, 276.

2 G. Ferey, Chem. Soc. Rev., 2008, 37, 191.

3 S. Horike, S. Shimomura and S. Kitagawa, Nat. Chem., 2009, 1, 695. 
4 J. B. DeCoste and G. W. Peterson, Chem. Rev., 2014, 114, 5695.

5 G. Odell Wood, Carbon, 1992, 30, 593.

6 G. W. Peterson, J. A. Rossin, P. B. Smith and G. W. Wagner, Carbon, 2010, 48, 81.

7 E. Barea, C. Montoro and J. A. R. Navarro, Chem. Soc. Rev., 2014, 43, 5419.

8 J. Guo, W. S. Xu, Y. L. Chen and A. C. Lua, J. Colloid Interface Sci., 2005, 281, 285.

9 D. Britt, H. Furukawa, B. Wang, T. G. Glover and O. M. Yaghi, Proc. Natl. Acad. Sci. U. S. A., 2009, 106, 20637.

10 J.-R. Li, R. J. Kuppler and H.-C. Zhou, Chem. Soc. Rev., 2009, 38, 1477.

11 T. M. McDonald, J. A. Mason, X. Kong, E. D. Bloch, D. Gygi, A. Dani, V. Crocella, F. Giordanino, S. O. Odoh, W. S. Drisdell, B. Vlaisavljevich, A. L. Dzubak, R. Poloni, S. K. Schnell, N. Planas, K. Lee, T. Pascal, L. F. Wan, D. Prendergast, J. B. Neaton, B. Smit, J. B. Kortright, L. Gagliardi, S. Bordiga, J. A. Reimer and J. R. Long, Nature, 2015, 519, 303.

12 J. M. Holcroft, K. J. Hartlieb, P. Z. Moghadam, J. G. Bell, G. Barin, D. P. Ferris, E. D. Bloch, M. M. Algaradah, M. S. Nassar, Y. Y. Botros, K. M. Thomas, J. R. Long, R. Q. Snurr and J. F. Stoddart, J. Am. Chem. Soc., 2015, 137, 5706.

13 D. Britt, D. Tranchemontagne and O. M. Yaghi, Proc. Natl. Acad. Sci. U. S. A., 2008, 105, 11623.

14 N. A. Khan, Z. Hasan and S. H. Jhung, J. Hazard. Mater., 2013, 244-245, 444.

15 T. Grant Glover, G. W. Peterson, B. J. Schindler, D. Britt and O. Yaghi, Chem. Eng. Sci., 2011, 66, 163.

16 K. C. Kim, P. Z. Moghadam, D. Fairen-Jimenez and R. Q. Snurr, Ind. Eng. Chem. Res., 2015, 54, 3257.

17 G. W. Peterson, J. B. DeCoste, F. Fatollahi-Fard and D. K. Britt, Ind. Eng. Chem. Res., 2013, 53, 701.

18 J. B. DeCoste, G. W. Peterson, B. J. Schindler, K. L. Killops, M. A. Browe and J. J. Mahle, J. Mater. Chem. A, 2013, 1, 11922.

19 L. Bellarosa, S. Calero and N. Lopez, Phys. Chem. Chem. Phys., 2012, 14, 7240.

20 J. Canivet, A. Fateeva, Y. Guo, B. Coasne and D. Farrusseng, Chem. Soc. Rev., 2014, 43, 5594.

21 S. S. Kaye, A. Dailly, O. M. Yaghi and J. R. Long, J. Am. Chem. Soc., 2007, 129, 14176.

22 J. B. Decoste, G. W. Peterson, M. W. Smith, C. A. Stone and C. R. Willis, J. Am. Chem. Soc., 2012, 134, 1486.

23 J. G. Nguyen and S. M. Cohen, J. Am. Chem. Soc., 2010, 132, 4560.

24 P. Z. Moghadam, P. Ghosh and R. Q. Snurr, J. Phys. Chem. C, 2015, 119, 3163.

25 P. Deria, J. E. Mondloch, E. Tylianakis, P. Ghosh, W. Bury, R. Q. Snurr, J. T. Hupp and O. K. Farha, J. Am. Chem. Soc., 2013, 135, 16801.

26 C. Yang, U. Kaipa, Q. Z. Mather, X. Wang, V. Nesterov, A. F. Venero and M. A. Omary, J. Am. Chem. Soc., 2011, 133, 18094.

27 H. Jasuja, N. C. Burtch, Y.-G. Huang, Y. Cai and K. S. Walton, Langmuir, 2012, 29, 633.
28 P. Ghosh, K. C. Kim and R. Q. Snurr, J. Phys. Chem. C, 2013, 118, 1102.

29 C. E. Wilmer, M. Leaf, C. Y. Lee, O. K. Farha, B. G. Hauser, J. T. Hupp and R. Q. Snurr, Nat. Chem., 2012, 4, 83.

30 D. Fairen-Jimenez, N. A. Seaton and T. Düren, Langmuir, 2010, 26, 14694.

31 P. Küsgens, M. Rose, I. Senkovska, H. Fröde, A. Henschel, S. Siegle and S. Kaskel, Microporous Mesoporous Mater., 2009, 120, 325.

32 C. Montoro, F. Linares, E. Quartapelle Procopio, I. Senkovska, S. Kaskel, S. Galli, N. Masciocchi, E. Barea and J. A. R. Navarro, J. Am. Chem. Soc., 2011, 133, 11888.

33 A. Comotti, S. Bracco, P. Sozzani, S. Horike, R. Matsuda, J. Chen, M. Takata, Y. Kubota and S. Kitagawa, J. Am. Chem. Soc., 2008, 130, 13664.

34 Q. Min Wang, D. Shen, M. Bülow, M. Ling Lau, S. Deng, F. R. Fitch, N. O. Lemcoff and J. Semanscin, Microporous Mesoporous Mater., 2002, 55, 217.

35 P. M. Schoenecker, C. G. Carson, H. Jasuja, C. J. J. Flemming and K. S. Walton, Ind. Eng. Chem. Res., 2012, 51, 6513.

36 N. C. Burtch, H. Jasuja and K. S. Walton, Chem. Rev., 2014, 114, 10575.

37 B. Widom, J. Chem. Phys., 1963, 39, 2808.

38 R. L. June, A. T. Bell and D. N. Theodorou, J. Phys. Chem. Lett., 1990, 94, 1508.

39 W. L. Jorgensen, J. Chandrasekhar, J. D. Madura, R. W. Impey and M. L. Klein, J. Chem. Phys., 1983, 79, 926.

40 L. Zhang and J. I. Siepmann, ChemPlusChem, 2010, 75, 577. 41 M. G. Martin and J. I. Siepmann, J. Phys. Chem. B, 1998, 102, 2569.

42 A. K. Rappe, C. J. Casewit, K. S. Colwell, W. A. Goddard and W. M. Skiff, J. Am. Chem. Soc., 1992, 114, 10024.

43 V. H. Dalvi, V. Srinivasan and P. J. Rossky, J. Phys. Chem. C, 2010, 114, 15553.

44 A. Ö. Yazaydın, R. Q. Snurr, T.-H. Park, K. Koh, J. Liu, M. D. LeVan, A. I. Benin, P. Jakubczak, M. Lanuza, D. B. Galloway, J. J. Low and R. R. Willis, J. Am. Chem. Soc., 2009, 131, 18198.

45 C. E. Wilmer, K. C. Kim and R. Q. Snurr, J. Phys. Chem. Lett., 2012, 3, 2506.

46 D. Frenkel and B. Smit, Understanding Molecular Simulation: From Algorithms to Applications (Computational Science), Academic Press, 2nd edn, 2001.

47 D. Dubbeldam, S. Calero, D. E. Ellis and R. Q. Snurr, Mol. Simul., 2016, 42, 81.

48 G. W. Peterson, G. W. Wagner, A. Balboa, J. Mahle, T. Sewell and C. J. Karwacki, J. Phys. Chem. C, 2009, 113, 13906.

49 G. W. Peterson, J. B. DeCoste, F. Fatollahi-Fard and D. K. Britt, Ind. Eng. Chem. Res., 2014, 53, 701.

50 L. Sarkisov, J. Phys. Chem. C, 2012, 116, 3025.

51 C. Yang, X. Wang and M. A. Omary, J. Am. Chem. Soc., 2007, 129, 15454.

52 H. Furukawa, F. Gándara, Y.-B. Zhang, J. Jiang, W. L. Queen, M. R. Hudson and O. M. Yaghi, J. Am. Chem. Soc., 2014, 136, 4369.

53 G. Akiyama, R. Matsuda, H. Sato, A. Hori, M. Takata and S. Kitagawa, Microporous Mesoporous Mater., 2012, 157, 89. 
54 V. Bon, I. Senkovska, M. S. Weiss and S. Kaskel, CrystEngComm, 2013, 15, 9572.

55 H. Reinsch, M. A. van der Veen, B. Gil, B. Marszalek, T. Verbiest, D. de Vos and N. Stock, Chem. Mater., 2013, 25, 17.

56 D.-A. Yang, H.-Y. Cho, J. Kim, S.-T. Yang and W.-S. Ahn, Energy Environ. Sci., 2012, 5, 6465.
57 Y. J. Colon and R. Q. Snurr, Chem. Soc. Rev., 2014, 43, 5735. 58 J. Masih, J. Chem. Pharm. Res., 2010, 2, 546.

59 K. Sumida, D. L. Rogow, J. A. Mason, T. M. McDonald, E. D. Bloch, Z. R. Herm, T.-H. Bae and J. R. Long, Chem. Rev., 2012, 112, 724.

60 H. Jasuja, G. W. Peterson, J. B. Decoste, M. A. Browe and K. S. Walton, Chem. Eng. Sci., 2015, 124, 118. 